\title{
An unusual case of ileo-uterine fistula discovered after full term normal delivery
}

\author{
Suneeta Singh$^{1}$, Sanjay Singh ${ }^{1 *}$, Sanjay Sharma ${ }^{2}$, Opjinder Singh Deepak ${ }^{3}$, Ahijit Banerjee ${ }^{4}$
}

\author{
${ }^{1}$ Department of Obstetrics and Gynecology, Base Hospital Delhi Cantt, New Delhi, India \\ ${ }^{2}$ Department of Surgery, Base Hospital Delhi Cantt, New Delhi, India \\ ${ }^{3}$ Department of Surgery, Command Hospital Udhampur, Jammu and Kashmir, India \\ ${ }^{4}$ Department of Anesthesia, Base Hospital Delhi Cantt, New Delhi, India
}

Received: 23 February 2020

Accepted: 27 March 2020

\section{*Correspondence:}

Dr. Sanjay Singh,

E-mail: drsanjaysingh@gmail.com

Copyright: (C) the author(s), publisher and licensee Medip Academy. This is an open-access article distributed under the terms of the Creative Commons Attribution Non-Commercial License, which permits unrestricted non-commercial use, distribution, and reproduction in any medium, provided the original work is properly cited.

\begin{abstract}
Entero-uterine fistula is a very rare type of fistula between the small intestine and the genital tract. They may result from obstetric or gynaecological procedures like post $\mathrm{D}$ and $\mathrm{C}$ perforation of the uterus. Timely detection and management reduce morbidity and mortality of the patients. We present a case of entero uterine fistula in a lady which was diagnosed on second postnatal day when she complained of passage of stool per vaginum. She had a previous history of mid trimester abortion followed by D and C after which she remained hospitalised for a week for pain abdomen. The diagnosis of this fistula was based on her symptoms and clinical evaluation of passage of intestinal content through the cervix and CT scan finding of gas in the abdomen. Intraoperatively an ileal loop was found adherent to the posterior wall of uterus with large fistula between bowel and the uterus. There was no intraperitoneal faecal contamination. Adherent small bowel was separated from the posterior wall of the uterus and fistula dismantled. Side to side functional end to end anastomosis was done using TLC 75 stapler. Repair of the uterine rent was also done. Post op recovery was uneventful.
\end{abstract}

Keywords: Curettage, Ileo-uterine fistula, Obstetric injury, Perforation

\section{INTRODUCTION}

A rare complication of curettage is uterine perforation which can get complicated by gut adhesion to the uterus and very rarely formation of entero-uterine fistula. Entero-uterine fistula is a very rare type of fistula between the small intestine and the genital tract. They may result from obstetric or gynaecological procedures, pelvic tumours, inflammatory disease and post D and C perforation of the uterus. ${ }^{1-3} \mathrm{~A}$ high index of suspicion is needed to diagnose this entity. CT and MRI are useful investigations to demonstrate the site and track of fistula. Surgical resection of fistula with primary anastomosis is curative in majority of the cases. Here, case present a case of entero uterine fistula with a brief review of this unusual and interesting case.

\section{CASE REPORT}

A 28-year-old P4L3A2 lady underwent full term normal delivery. On second postnatal day she complained of passage of stool per vaginum. In her previous pregnancy, she gave history of intra uterine demise at $5^{\text {th }}$ month. She had incomplete abortion at home and in view of excessive vaginal bleeding she was brought to the hospital for which she underwent suction and evacuation ( $S$ and $E$ ) followed by curettage. She had to remain hospitalized for ten days after the procedure for fever and 
pain abdomen. She also had history of pulmonary Kochs for which she took ATT almost ten years back.

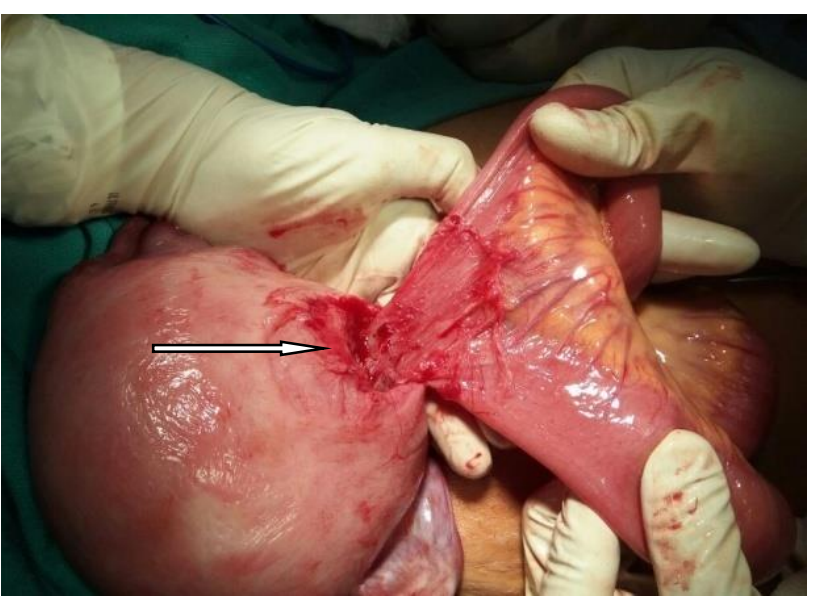

Figure 1: On laparotomy ileal loop found adherent to the posterior wall of uterus (white arrow).

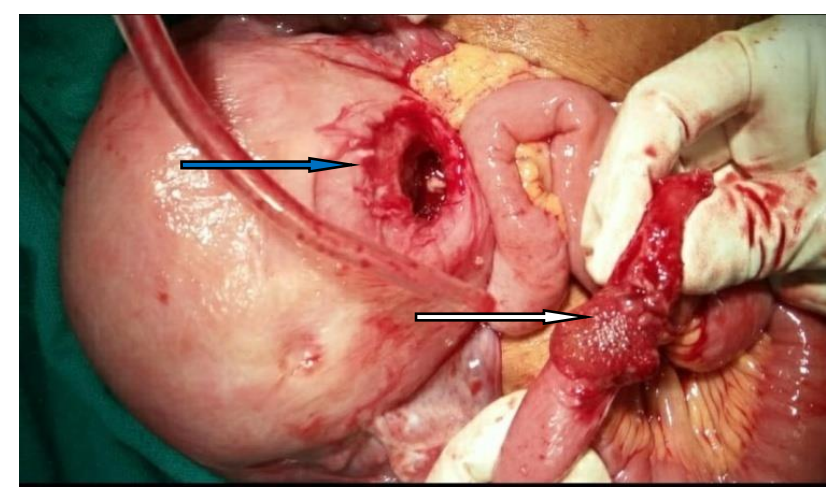

Figure 2: On laparotomy a rent was noted on posterior wall of uterus (blue arrow) communicating with a rent in the ileum (white arrow).

The antenatal and intrapartum period of the present pregnancy was uneventful. Her general and systemic examinations were essentially normal. On local examination vagina was healthy with no rent visible. However, the intestinal content was seen coming through the external os of the cervix. Further imaging (by computed tomography), showed few gas shadows in abdomen suggestive of breach in the gastrointestinal tract. We had no magnetic resonance imaging (MRI) facility.

A laparotomy was performed. Intraoperatively an Ileal loop was found adherent to the posterior wall of uterus (Figure 1) with large fistulous tract between bowel and the uterus. 3-4 cm rent was seen on posterior wall of uterus (Figure 2). There was no intraperitoneal faecal contamination. Adherent small bowel was separated from the posterior wall of the uterus and fistula dismantled. Side to side functional end to end anastomosis was done using TLC 75 stapler. Repair of the uterine rent was also done. Thorough Peritoneal wash was given and $28 \mathrm{~F}$ intraperitoneal drain was put. Abdomen was closed in layers.

Post op she had fever of $1010 \mathrm{~F}$ for two days. She responded well to the antibiotics and they were stopped after 5 days. The intraperitoneal drain was removed after 3 days. She was started on oral fluids from second postoperative day followed by soft diet started from third post op day. She was discharged to home on the 10th post op day.

\section{DISCUSSION}

Fistulous communication between the uterus and gastrointestinal tract is very rare. The entero-uterine fistulas are generally found between the fundus of the uterus and the loops of small bowel. These fistulas have been described in cases of gynecological cancers and cases of ulcerative colitis, and rarely following D and C procedures. ${ }^{1-3}$ The fistula in our case most likely resulted from perforation of the uterus at the time of suction and curettage in the previous pregnancy. There is a case report of utero-rectal fistula manifesting during second trimester with massive GI bleeding. ${ }^{4}$

Uterine perforation is a dreaded but known complication of $\mathrm{D}$ and $\mathrm{C}$ procedure. Amarin and Badria described an incidence of $0.2 \%$ in a series of 11,914 women when D and $\mathrm{C}$ was performed for retained products of conception..$^{5}$ Darney et al published a series of 15 cases of post $\mathrm{D}$ and $\mathrm{C}$ uterine perforation, with two requiring bowel resection and two undergoing hysterectomy. ${ }^{6}$

In 1933 Danforth and case published a series of 33 cases of entero-uterine fistula from literature covering the preceding 200 years. $^{7}$ The common causes in their series were traumatic or spontaneous rupture of uterus with entrance of loop of intestine, peritonitis with abscess formation and simultaneous rupture of abscess into the uterus and the bowel, carcinoma of uterus with ulceration and necrosis resulting in fistula formation. A fourth cause was added by Hawkes as perforation of uterus and bowel while doing curettage. ${ }^{8}$ Martin et al published the largest series entero-uterine fistula in 1956 in which he described 80 cases, 40 of which followed obstetric injury, 17 resulted from inflammatory processes, 12 following curettage and 9 related to carcinoma. ${ }^{9}$ Other causes of these fistulas include chronic inflammatory bowel disease, complication of diverticulitis, neoplasm, endometriosis. ${ }^{1,2}$

The diagnosis is based on history and finding of faecal discharge per vaginum. It relies on high degree of suspicion and careful physical examination. In post-natal case the first diagnosis which comes to mind is rectovaginal fistula. But pelvic examination in the cases of entero uterine fistula will reveal an intact vaginal wall and the intestinal content will be seen coming out from the cervical os. In this case too vaginal wall was intact and intestinal content was seen coming out through' 
external os. Magnetic resonance imaging (MRI) helps in delineating the fistula and detecting any associated extraluminal disease. ${ }^{10}$

A careful and meticulously adopted technique reduces the risk of uterine perforation during $\mathrm{D}$ and $\mathrm{C}$. The management of the fistula depends on the nature of the fistula, the nutritional status of the patient and the presence of associated disease. Surgical therapy is usually advocated that includes resection of the involved bowel with primary anastomosis and closure of the uterine defect. ${ }^{10-12}$ Early presentation with minimal contamination particularly with small bowel involvement has reported to have better outcome. ${ }^{13}$ Rarely, temporary ileostomy with prolonged nutritional support may be required. ${ }^{11,12}$ In this case we did the surgical resection of the fistula followed by end to end anastomosis of the ileum.

\section{CONCLUSION}

Early diagnosis and prompt intervention on the suspicion on inadvertent uterine perforation will prevent significant morbidity. Closure of the uterine perforation should be undertaken to prevent fistula formation. In case of enterouterine fistula, surgical resection of the fistula with primary anastomosis is curative in the majority of cases.

Funding: No funding sources Conflict of interest: None declared

Ethical approval: Not required

\section{REFERENCES}

1. Taxonera C, Barreiro-de-Acosta M, Bastida G, Martinez-Gonzalez J, Merino O, García-Sánchez V, et al. Outcomes of medical and surgical therapy for entero-urinary fistulas in Crohn's disease. Journal of Crohn's and Colitis. 2016;10(6):657-62.

2. Clemente N, Alessandrini L, Giorda G, Sopracordevole F. Enterouterine fistula as initial presentation of advanced endometrial cancer: description of a rare case and review of the literature. J Clin Exp Oncol. 2016;5(6):2.

3. Shehata A, Hussein N, El Halwagy A, El Gergawy A, Khairallah M. Ileo-uterine fistula in a degenerated posterior wall fibroid after Caesarean section. Clin Exp Reprod Med. 2016;43(1):51-3.

4. Dasari P. Uterorectal fistula manifesting during second trimester of pregnancy as massive lower gastrointestinal bleeding: is it a menace of MTP?. Inter J Cur Res Rev. 2015;7(13):25.

5. Amarin ZO, Badria LF. A survey of uterine perforation following dilatation and curettage or evacuation of retained products of conception. Arch Gynecol Obstet. 2005;271(3):203-6.

6. Darney PD, Atkinson E, Hirabayashi K. Uterine perforation during second-trimester abortion by cervical dilation and instrumental extraction: a review of 15 cases. Obstet Gynecol. 2009:75(3):4414

7. Danforth WC, Case JT. Entero-uterine fistula with a review of the literature and report of a case studied radiologically. Am J Obst Gynecol. 1933;25:300-30.

8. Hawkes SZ. Enterouterine fistula; with a review of the literature and report of an unusual case. Am J Obstet Gynecol. 1946;52:150-3.

9. Martin DH, Hixson $\mathrm{CH}$, Wislon EC. Enterouterine fistula; review; report of an unusual case. Obstet Gynecol. 1956;7(4):466-9.

10. McFarlane ME, Plummer JM, Remy T, Christie L, Laws D, Richards H, et al. Jejunouterine fistula: a case report. Gynecol Surg. 2008;5(2):173.

11. Lloyd DA, Gabe SM, Windsor AC. Nutrition and management of enterocutaneous fistula. Br J Surg. 2006;93(9):1045-55.

12. Hjern F, Goldberg SM, Johansson C, Parker SC, Mellgren A. Management of diverticular fistulae to the female genital tract. Colorectal Dis. 2007;9(5):438-42.

13. Gupta S, Banerjee K, Bhardwaj DN, Dogra TD. Maternal death and induced abortion: a critical analysis. J Family Welfare. 2000;46(1):57-60.

Cite this article as: Singh $\mathrm{S}$, Singh $\mathrm{S}$, Sharma $\mathrm{S}$, Deepak OS, Banerjee A. An unusual case of ileouterine fistula discovered after full term normal delivery. Int J Reprod Contracept Obstet Gynecol 2020;9:2177-9. 